\title{
The identification of PM 1-322 as a new young high-density planetary nebulae ${ }^{\star}$
}

\author{
C. B. Pereira ${ }^{1}$ and L. F. Miranda ${ }^{2}$ \\ 1 Observatório Nacional-MCT, Rua José Cristino 77, CEP 20921-400, São Cristóvão, Rio de Janeiro-RJ, Brazil \\ e-mail: claudio@on.br \\ ${ }^{2}$ Instituto de Astrofísica de Andalucía, CSIC, Apdo.3004, 18080 Granada, Spain
}

Received 20 October 2004 / Accepted 30 November 2004

\begin{abstract}
We report spectroscopic observations of a new planetary nebula PM 1-322, which has not yet been identified. This object was found during a northern survey of post-AGB and planetary nebulae candidates selected from García-Lario et al. (1997, A\&AS, 126, 479) and Preite-Martinez (1988, A\&AS, 76, 317). Here we present the main spectroscopic features including a list of the intensities of the identified emission lines. The main spectral characteristic of PM 1-322 is the presence of a very strong $[\mathrm{O} \mathrm{III}] \lambda 4363$ emission line $(\mathrm{I}[\mathrm{OIII}] 4363 / \mathrm{H} \beta \simeq 120)$ indicating high electron densities $\left(\log N_{\mathrm{e}}>6.0\right)$. A comparison with similar objects strongly suggests that PM 1-322 is a very young planetary nebula, although the posibility of a symbiotic nature can not be ruled out.
\end{abstract}

Key words. planetary nebulae: individual: PM 1-322

\section{Introduction}

PM 1-322 (=IRAS 20124+1154) was first proposed as a planetary nebula (PN) candidate by Preite-Martinez (1988) on the basis of its infrared colours, proximity to the galactic equator and quality of the IRAS detection. PM 1-322 was selected for observation in the framework of our low resolution spectroscopy study of a large sample of Preite-Martinez's (1988) PN candidates as well as from García-Larios et al's (1997) transition objects (AGB and post-AGB stars to proto-PNe and $\mathrm{PNe}$ ). Previous results of this kind of investigation led us to the discovery of a post-AGB star with a WR-spectrum, GLMP 160 (Pereira \& Machado 2003).

The spectroscopic analysis of PM 1-322 indicates that this object is a compact PN with the high electron density typical of young objects. In this paper we present a discussion of the analysis of the spectroscopic observations and we comment on the criteria used to classify PM 1-322 as a young PN. We also compare it with other PNe previously classified as young highdensity PNe.

\section{Observations and reduction}

Spectroscopic observations were performed with CAFOS (Calar Alto Faint Object Spectrograph) attached to the $2.2 \mathrm{~m}$

* Based on observations collected at the Centro Astronomico Hispano Aleman (CAHA) at Calar Alto, operated jointly by the MaxPlanck Institut für Astronomie and the Instituto de Astrofísica de Andalucía (CSIC). telescope of the Calar Alto Observatory (Almeria, Spain). The detector was a SITe CCD with 2048 pixels in the spectral direction. The observations were done using the grisms $B-100$ and $R-100$ covering respectively the spectral range 3200 $5800 \AA$ and 5900-9000 A. The resolution obtained with both grisms is $2 \AA /$ pixel and the slit orientation was north-south. The resolution was enough to separate [O III] $\lambda 4363$ from $\mathrm{H} \gamma$ and [N II] $\lambda \lambda \lambda 6548,84$ from $\mathrm{H} \alpha$. The observations were done on August 26, 2004. The slit width was 4.'0 and the exposure time for both grisms was $2400 \mathrm{~s}$.

The spectra were reduced using standard IRAF tasks, from bias subtraction and flat-field correction, through spectral extraction and wavelength and flux calibration. Spectrophotometric standards from Oke (1974) and Massey et al. (1988) were also observed.

The flux of the emission lines were measured by the conventional method, adjusting a gaussian function to the line profile, thereby obtaining the intensity, the central wavelength and the line width at half power level. Uncertainties in the line intensities come mainly from the position of the underlying continuum. We estimate the flux error to be about $20 \%$ for the weak lines (line fluxes $\approx 10$ on the scale of $\mathrm{H} \beta=100$ ) and about $10 \%$ for stronger lines.

A finding chart of the object is present in Fig. 1. This chart is an image from POSS2/UKSTU in the red band. The equatorial coordinates of PM 1-322 are $\alpha(2000.0)=20^{\mathrm{h}} 14^{\mathrm{m}} 51^{\mathrm{s}}$ and $\delta(2000.0)=+12^{\circ} 03^{\prime} 50^{\prime \prime}$. The $V$ magnitude was derived by convolving the calibrated spectrum with a $V$ filter profile, giving 16.8 . 


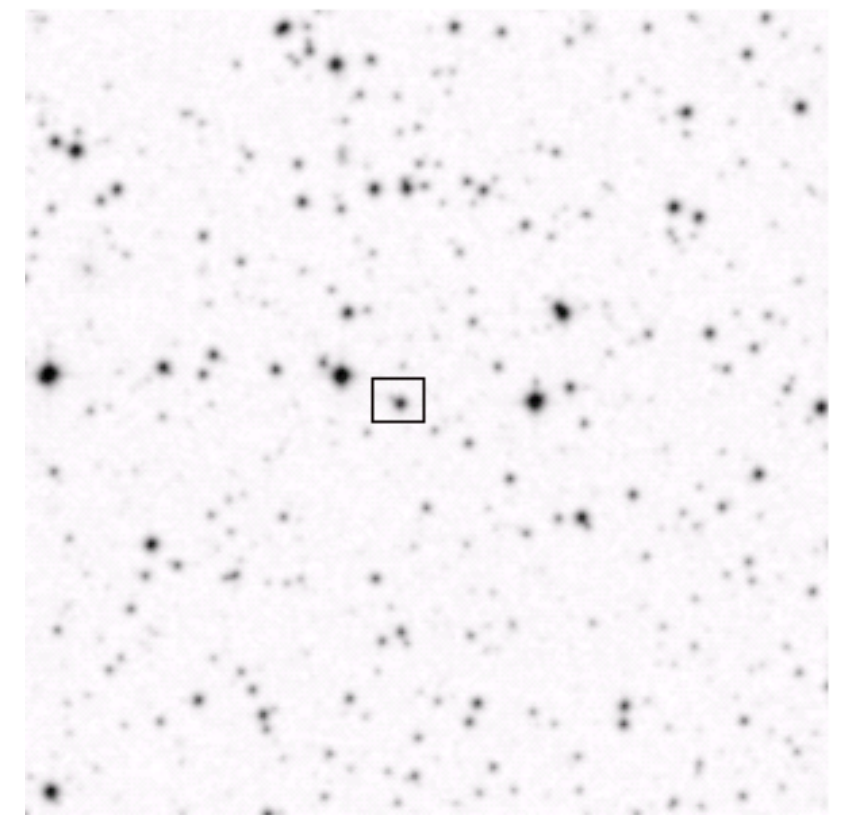

Fig. 1. Finding chart of the region around PM 1-322. PM 1-322 is at the center of the field. The field has dimensions of $7^{\prime} \times 7^{\prime}$. North is at the top and east is to the left.

\section{Results}

Figure 2 shows the flux calibrated spectra of PM 1-322 in the two observed spectral regions. The spectrum of PM 1-322 presents emission lines typical of a PN, such as the forbidden lines of [O III] $\lambda \lambda 4959,5007,[\mathrm{~N}$ II] $\lambda \lambda 6548,84$ and [S III] $\lambda \lambda$ 9069,9532 as well as He I, Balmer and Paschen recombination lines. Table 1 presents the identified emission lines and their observed intensities.

In low-density ionized nebulae such as $\mathrm{HII}$ regions and most planetary nebulae the usual procedure to obtain the amount of interstellar reddening towards the object consists in to comparing the observed Balmer line ratios with the theoretical values calculated under case B conditions. However, in high-density objects such as symbiotic stars and in highdensity planetary nebula, which is the case of PNe such as PM 1-322, Balmer line ratios indicate that self-absorption effects are present, thus making a direct application of this method difficult. In brief, self-absorption in Balmer lines occurs when the nebula is completely opaque in Lyman lines and also partially opaque in Balmer series. If the nebula is opaque to the $\mathrm{H} \beta$ line, the photons will vanish completely via cascade transitions with consequent emission of $\mathrm{P} \alpha, \mathrm{H} \alpha$ and $\mathrm{Ly} \alpha$. As a result, the number of $\mathrm{H} \beta$ photons from the nebula will decrease while the number of $\mathrm{H} \alpha$ will increase leading an increase of the $\mathrm{H} \alpha / \mathrm{H} \beta$ ratio in comparison with pure case $\mathrm{B}$ recombination. In order to overcome this difficult, the reddening can be estimated using the self-absorption calculations made by Capriotti (1964a,b). Using his results, the reddening can be obtained, by plotting the observed Balmer line ratios above mentioned in $\mathrm{H} \beta / \mathrm{H} \gamma$ vs. $\mathrm{H} \alpha / \mathrm{H} \beta$ plane and moving these points parallel to the reddening line crossing the theoretical curve, thus determining the reddening as well as the optical depth in $\mathrm{H} \alpha$. Using this procedure and from the observed line ratios $\mathrm{H} \alpha / \mathrm{H} \beta$ and $\mathrm{H} \gamma / \mathrm{H} \beta$ we obtain $E(B-V)=0.17$. It was assumed that the reddening law can be represented by the standard interstellar extinction curve $f(\lambda)$, (Seaton 1979).

The dereddened line intensity ratios of [O III] $(4959+5007) / 4363$, [S III] $(9532+9069) / 6312$ and [N II] $(6548+6584) / 5754$ for PM 1-332 are exceptionally low for a PN. The ratios involving these elements are, respectively, $6.3,8.0$ and 2.3. This indicates collisional de-excitation of the uppermost level for $\lambda \lambda(4959+5007), \lambda \lambda(6548+6584)$ and $\lambda \lambda(9069+9512)$. Under these conditions these nebular line ratios are useful density indicators. Assuming $T_{\mathrm{e}}=12000$, we have $\log N_{\mathrm{e}}([\mathrm{O}$ III $]), \log N_{\mathrm{e}}([\mathrm{S} \mathrm{III}])$ and $\log N_{\mathrm{e}}$ ([N II $\left.]\right)$ equal to, 6.54, 6.10 and 6.00, respectively. Similar densities are also found in symbiotic stars (Pereira et al. 1998; Schmid \& Schild 1990; Gutiérrez-Moreno \& Moreno 1996); and in the very young planetary nebulae Hen 2-57 (Kingsburgh \& Barlow 1994, hereafter KB94), Hen 2-35 (Corradi 1995) and IC 4997 (Hyung et al. 1994).

\section{Discussion}

The most interesting aspect of the spectrum of PM 1-322 is the relative strength of $\lambda 4363$ to $\mathrm{H} \beta(I(4363)=118.0$, Table $1)$. This relative line intensity is usually seen in the D- and D'-type symbiotic stars (Pereira et al. 1998; Pereira 1995; Gutiérrez-Moreno \& Moreno 1996). In order to better understand the nature of PM 1-322 we will investigate the position of this peculiar object in the diagnostic diagram of Gutiérrez-Moreno et al. (1995). This diagram uses the relative line strength $5007 / \mathrm{H} \beta$ versus $4363 / \mathrm{H} \gamma$. Figure 3 shows such a diagram. Since symbiotic stars are characterized by their excitation and density conditions, the relation between $\lambda \lambda 5007$ and 4363 is a good measure of their physical conditions. In $\mathrm{PNe}$ the ratio between these two lines may range from 50 to 200 (KB94).

In Figure 3 we see that symbiotic stars and PNe in most cases are clearly distinguished according to their relative line intensities. For electron densities higher than $N_{\mathrm{e}}=10^{5} \mathrm{~cm}^{-3}$, which is the case for all symbiotics, collisional de-excitation becomes important and thus contributes more strongly to the depopulation of the middle $\left({ }^{1} D_{2}\right)$ level than to the depopulation of the upper $\left({ }^{1} \mathrm{~S}_{0}\right)$ level. This results in the weakening of the emission lines originating in the middle level $\left({ }^{1} \mathrm{D} \rightarrow{ }^{3} \mathrm{P}\right)$ with respect to the line emitted from the upper level $\left({ }^{1} \mathrm{~S} \rightarrow{ }^{1} \mathrm{D}\right)$ from which the emission line $\lambda 4363$ originates. Figure 3 clearly shows these effects which help us to see the main differences between PNe (data from KB94) and symbiotic stars (S-type from Gutiérrez-Moreno et al. 1999; D- and D'type from Schmid \& Schild 1990; Pereira 1995; Pereira et al. 1998; Schmid \& Nussbaumer 1993, and Gutiérrez-Moreno \& Moreno 1996). However, we see that PM 1-322 (asterisk) occupies the same region as the symbiotics. But since in PM 1-322 we do not observe any absorption line or TiO band nor an increase of the continuum towards the red, which would indicate the presence of a companion, it is unlikely to be a binary symbiotic system The most likely explanation of why the position of 

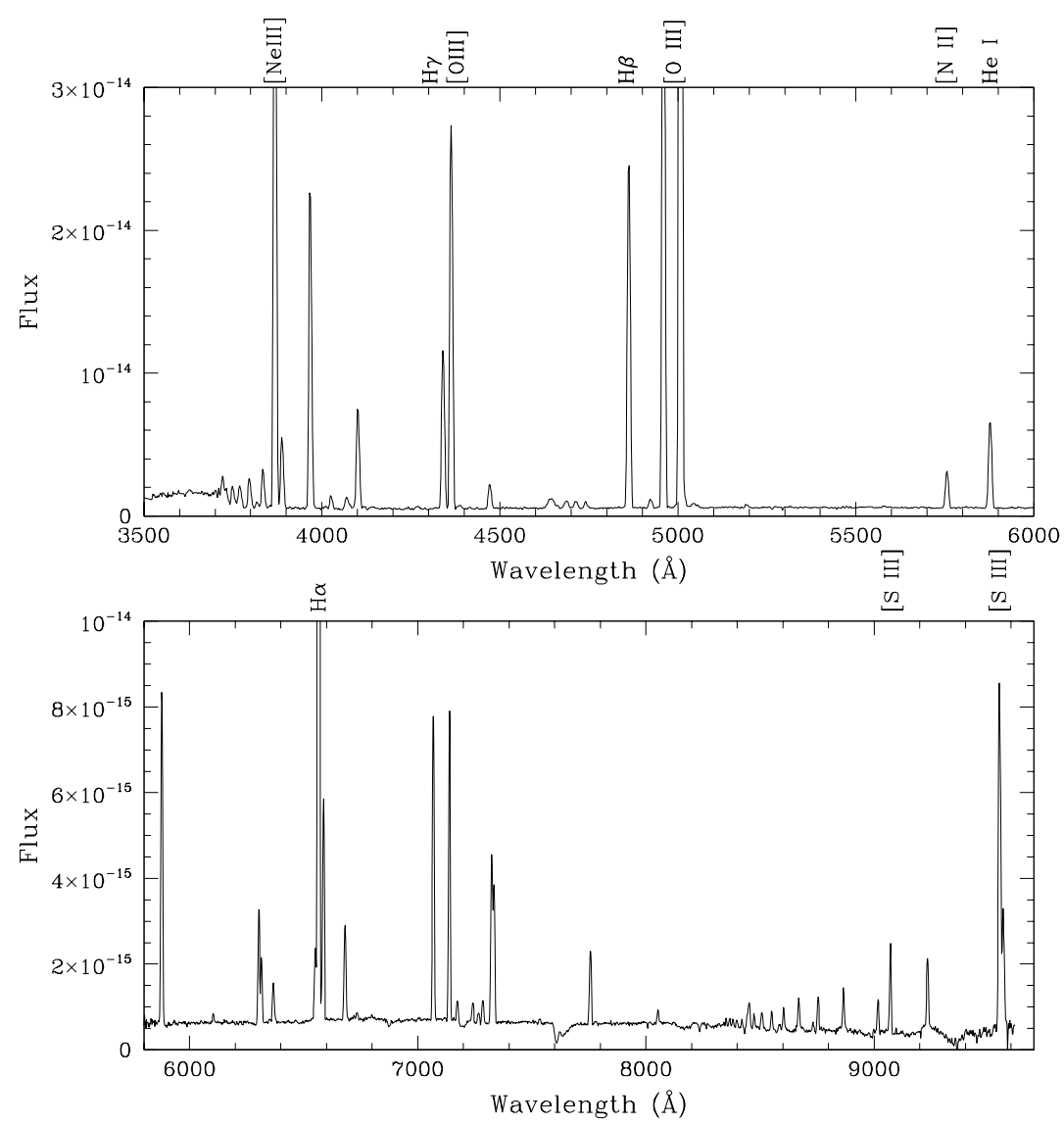

Fig. 2. Blue (top) and red (bottom) spectrum of PM 1-322. Notice the strength of [O III] $] 4363$.

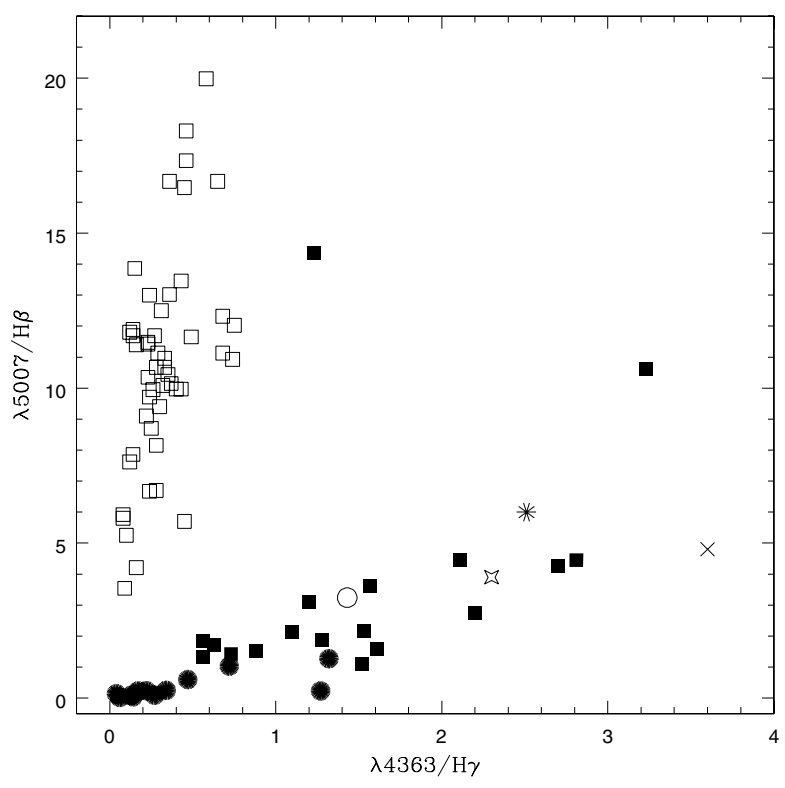

Fig. 3. Position of PNe, D'-, D- and S-type symbiotics, PM 1-322, IC 4997, Hen $2-25$ and Hen $2-57$ in the $\lambda 5007 / \mathrm{H} \beta-\lambda 4363 / \mathrm{H} \gamma$ plane. Open squares: planetary-nebulae (Kingsburgh \& Barlow 1994); filled square points: D- and $\mathrm{D}^{\prime}$-type symbiotics (Schmid \& Nussbaumer 1993; Gutiérrez-Moreno \& Moreno 1996; Schmid \& Schild 1990; Pereira 1995; Pereira et al. 1998); filled circles: S-type symbiotics (Pereira 1995; Gutiérrez-Moreno et al. 1999; Schmid \& Schild 1990); open circle: Hen 2-57 (Kingsburgh \& Barlow 1994); asterisk: PM 1-322 (This work); star: IC 4997 (Hyung et al. 1994); cross: Hen 2-25 (Corradi 1995)
PM 1-322 in this diagram is the same as that of the symbiotics, is that it is a young high-density planetary nebula.

Figure 3 also shows the positions of other objects already classified as young high-density planetary-nebulae; Hen 2-57 from KB94 and IC 4997 from Hyung et al. (1994). The other object, Hen 2-25 from Corradi (1995) might be a symbiotic or a PNe. Although the optical spectrum of Hen 2-25 is typical of a PNe (flat continuum plus emission lines) the possibility of its being a symbiotic cannot be totally disregarded; some symbiotics may present in their emission spectra several iron emission-lines (Hen 3-1674 and V2929 Sgr; Munari \& Zwitter 2002), which is not the case for PM 1-322. It is also interesting to note that two of the young PNe mentioned here (Hen 2-57 and Hen 2-25) are bipolar PNe (Corradi 1995; KB94). In the case of PM 1-322, images in $\mathrm{H} \alpha$, [N II] and [O III] (not presented here) with a spatial resolution of $\simeq 1.8$ arcsec taken at the Observatorio of Sierra Nevada show a stellar object without traces of internal structure.

Although we have presented strong evidence that PM 1-322 might be a young planetary-nebula, it should be kept in mind that there is a slight possibility that it is a symbiotic system. In fact in three symbiotic stars, H 1-36, Hen 2-104 and Hen 2-106, the optical spectra do not present strong evidence of any absorption line and/or TiO bands. In these systems the cool star is very weak so that its presence could only be detected in the infrared. In these cases their symbiotic nature is revealed due by the presence of the $\lambda 6830$ band (Schmid 1989) in Hen 2-106 (Munari \& Zwitter 2002) and in H 1-36 (Pereira et al. 1998). 
Table 1. Observed emission line fluxes relative to $H \beta=100$.

\begin{tabular}{|c|c|c|}
\hline Wavelength & Identification & $F(\lambda)$ \\
\hline 3771 & H 11 & 7.8 \\
\hline 3798 & H 10 & 8.9 \\
\hline 3835 & H9 & 10.7 \\
\hline 3869 & [Ne III] & 260.5 \\
\hline 3889 & $\begin{array}{l}\mathrm{He} \text { I } \\
\mathrm{H} 8\end{array}$ & 27.9 \\
\hline 3968 & $\begin{array}{c}{[\mathrm{Ne} \text { III] }} \\
\mathrm{H} \epsilon\end{array}$ & 102.8 \\
\hline 4026 & He I & 4.3 \\
\hline 4068 & [S II $]$ & 3.7 \\
\hline 4076 & [S II] & 1.3 \\
\hline 4101 & $\mathrm{H} \delta$ & 29.8 \\
\hline 4340 & $\mathrm{H} \gamma$ & 47.1 \\
\hline 4363 & [O III] & 118.2 \\
\hline 4471 & $\mathrm{He} \mathrm{I}$ & 7.9 \\
\hline 4640 & N III & 7.1 \\
\hline 4686 & He II & 3.5 \\
\hline 4922 & He I & 2.9 \\
\hline 4959 & [O III] & 201.6 \\
\hline 5007 & [O III] & 612.9 \\
\hline 5047 & $\mathrm{He} \mathrm{I}$ & 21.0 \\
\hline 5056 & Si II & 1.0 \\
\hline 5754 & {$[\mathrm{~N}$ II $]$} & 11.3 \\
\hline 5876 & $\mathrm{He} \mathrm{I}$ & 28.3 \\
\hline 6300 & {$[\mathrm{OI}]$} & 10.7 \\
\hline 6312 & {$\left[\mathrm{~S}^{*} \mathrm{III}\right]$} & 5.7 \\
\hline 6363 & {$[\mathrm{OI}]$} & 3.8 \\
\hline 6548 & [N II] & 8.3 \\
\hline 6563 & $\mathrm{H} \alpha$ & 393.2 \\
\hline 6584 & {$[\mathrm{~N}$ II $]$} & 20.2 \\
\hline 6678 & He I & 8.6 \\
\hline 6717 & {$[\mathrm{~S}$ II $]$} & 0.4: \\
\hline 6731 & [S II] & 0.7 \\
\hline 7065 & He I & 27.2 \\
\hline 7135 & [Ar III] & 27.7 \\
\hline 7170 & [Ar IV] & 1.8 \\
\hline 7237 & [Ar IV] & 2.1 \\
\hline 7263 & [Ar IV] & 1.2 \\
\hline 7281 & He I & 2.3 \\
\hline 7320 & [O II $]$ & 18.4 \\
\hline 7330 & [O II] & 10.2 \\
\hline 7751 & [Ar III] & 7.6 \\
\hline 8046 & [Cl IV] & 1.5 \\
\hline 8446 & O I & 1.3 \\
\hline 8467 & P 17 & 1.3 \\
\hline 8502 & P 16 & 1.5 \\
\hline 8545 & P 15 & 1.9 \\
\hline 8498 & P 14 & 2.3 \\
\hline 8665 & $\mathrm{P} 13$ & 2.9 \\
\hline 8727 & {$[\mathrm{CI}]$} & 1.2 \\
\hline 8750 & $\mathrm{P} 12$ & 3.3 \\
\hline 8862 & $\mathrm{P} 11$ & 4.1 \\
\hline 9014 & P 10 & 3.9 \\
\hline 9069 & [S IIII] & 8.7 \\
\hline 9229 & P9 & 7.8 \\
\hline 9532 & [S III $]$ & 46.4 \\
\hline 9546 & $\mathrm{P} 8$ & 20.4 \\
\hline$F(\mathrm{H} \beta)\left[\mathrm{erg} / \mathrm{cm}^{2} \mathrm{~s}\right]$ & $2.5 \times 10^{-13}$ & 100.0 \\
\hline
\end{tabular}

\section{Conclusions}

New young PNe candidates have been found either through a radio continuum survey (Aaquist \& Kwok 1990) or through a survey using the IRAS color-color diagram (Pottasch et al. 1990). In the first case the planetary-nebulae that have a high radio surface brightness temperature indicative of young planetary nebulae can be compact objects either because they are very distant (having a star-like appearance) or because they are physically small. In the second case the position of the object in the IRAS color-color diagram indicates the object as a "potential PNe" (Pottasch et al. 1990). So, an important aspect in confirming or disregarding the nature of the object is to observe it either spectroscopically or by imaging. Here by using spectroscopic observations we confirmed PM 1-322 as a PN, after selecting this object from the IRAS survey of Preite-Martinez (1988). Although the spectral characteristics strongly suggests that PM 1-322 is a young PN, its high electron density and the position in the $4363 / \mathrm{H} \gamma$ vs. $5007 / \mathrm{H} \beta$ diagram do not allow us to totally rule out the possibility of its being a symbiotic nature.

Acknowledgements. We are very grateful to the staff of the Calar Alto Observatory by their excellent assistance during the observations. This work has been supported partially by grant AYA2002-00376 of the Spanish MEC (cofunded by FEDER funds). We also thank the referee for the comments that have helped us to improve the presentation of the paper.

\section{References}

Aaquist, O. B., \& Kwok, S. 1990, A\&AS, 84, 229

Capriotti, E. R. 1964a, ApJ, 139, 225

Capriotti, E. R. 1964b, ApJ, 140, 632

Corradi, R. 1995, MNRAS, 276, 521

García-Lario, P., Manchado, P., Pych, W., Pottasch, S. R. 1997, A\&AS, 126, 479

Gutiérrez-Moreno, A., Moreno, H., \& Cortés, G. 1995, PASP, 107, 462

Gutiérrez-Moreno, A., \& Moreno, H. 1996, PASP, 108, 972

Gutiérrez-Moreno, A., Moreno, H., \& Costa, E. 1999, PASP, 111, 571

Hyung, S., Aller, L. H., \& Feibelman, W. A. 1994, ApJS, 93, 465

Kingsburgh, R. L., \& Barlow, M. J. 1994, MNRAS, 271, 257 (KB94)

Massey, P., Strobel, K., Barnes, J. V., Anderson, E. 1988, ApJ, 328, 315

Munari, U., \& Zwitter, T. 2002, A\&A, 383, 188

Oke, J. B. 1974, ApJS, 27, 21

Pereira, C. B. 1995, A\&AS, 111, 471

Pereira, C. B., Landaberry, S. J. C., \& Junqueira, S. 1998, A\&A, 333, 658

Pereira, C. B., de Araújo, F. X., \& Landaberry, S. J. C. 1999, MNRAS, 309,1074

Pereira, C. B., \& Machado, M. A. D. 2003, A\&A, 407, 311

Pottasch, S. R., Ratag, M. A., \& Olling, R. 1990, in From Miras to planetary-nebulae: Which path for stellar evolution? Proc. International Coll. (Montpellier: Editions Frontières), 381

Osterbrock, D. E. 1974, Astrophysics of Gaseous Nebulae (San Francisco: W. H. Freeman)

Preite-Martinez, A. 1988, A\&AS, 76, 317

Schmid, H. M. 1989, A\&A, 211, L31

Schmid, H. M., \& Schild, H. 1990, MNRAS, 246, 84

Schmid, H. M., \& Nussbaumer, H. 1993, A\&A, 268, 159

Seaton, M. J. 1979, MNRAS, 187, 73 